\title{
Coronavirus: epidemiology, genome replication and the interactions with their hosts
}

\author{
Zheng-Li Shi $^{1 \bowtie}$, Deyin Guo ${ }^{2 \bowtie}$, Peter J. M. Rottier ${ }^{3 \bowtie}$
}

1. Key Laboratory of Special Pathogens, Wuhan Institute of Virology, Chinese Academy of Sciences, Wuhan 430071, China

2. State Key Laboratory of Virology, College of Life Sciences, Wuhan University, Wuhan 430070, China

3. Virology Division, Department of Infectious Diseases and Immunology, Faculty of Veterinary Medicine, Utrecht University, Utrecht 3584 CL, Netherlands

This special issue of the journal is dedicated to the recent progress on coronaviruses and covers the topics of viral epidemiology, virus replication and the interactions between the coronaviruses and their hosts.

Members of the family Coronaviridae infect a wide range of vertebrates and humans. They usually cause respiratory, gastrointestinal, hepatic and/or central nervous system diseases of humans, livestock and wild animals. Prior to the outbreak of severe acute respiratory syndrome (SARS), only two coronaviruses, the human coronaviruses hCoV-229E and hCoV-OC43, were known to infect humans. Following the SARS outbreak, four additional coronaviruses have been discovered in humans including the SARS coronavirus (SARS-CoV), human coronavirus hCoV-NL63, human coronavirus hCoV-HKU1 and Middle East respiratory syndrome coronavirus (MERS-CoV). SARS$\mathrm{CoV}$ and MERS-CoV are highly pathogenic in humans and cause severe acute respiratory distress with a high rate of mortality. Remarkably, both viruses are believed to have originated from bats. In this special issue, Yip et al. report on a six-year longitudinal surveillance of human coronavirus infections in Hong Kong that provides new insight into the epidemiology of coronaviruses (Yip et al., 2016). Niu et al. present the development of a new two-tube multiplex real-time reverse transcription PCR to detect six human coronaviruses (Niu et al., 2016). Three articles, authored by $\mathrm{Ge}$ et al., Xu et al. and Wang et al., describe different discoveries on more diverse bat coronaviruses including SARS-like coronaviruses, highlighting the importance of continued further research on bat-related coronaviruses (Ge et al., 2016; Wang et al., 2016; $\mathrm{Xu}$ et al., 2016). And Wang et al. compared the lentiviruses pseudotyped with S proteins from coronaviruses and cell tropisms of two porcine coronaviruses (Wang et al., 2016).

The genomes of coronaviruses consist of a positive and singlestranded RNA genome of about 30 kilo bases, the largest genomes known among RNA viruses. Twothirds of the coronavirus genome at the $5^{\prime}$ terminus encodes viral replicase/transcriptase functions which are involved in virus replication, while one-third at the $3^{\prime}$ terminus encodes viral structural proteins and virus group-specific accessory proteins. Functional and mechanistic studies on these viral proteins are pivotal for drug screening and vaccine development against the diseases caused by coronaviruses. In this issue, Chen and Guo give an overview of recent progresses on molecular mechanisms of coronavirus RNA capping and methylation (Chen et al., 2016). Wang et al., summarize the recent advances in biological and structural studies, together with the development of inhibitors targeting coronavirus proteases (Wang et al., 2016).

Zoonotic coronaviruses are becoming a global concern due to the emergence of the two highly pathogenic coronaviruses, SARS-CoV and MERS-CoV. Infections by these emerging human coronaviruses are characterized by less robust interferon production. In this issue, the review authored by Wong et al. focuses on the interactions between host innate antiviral immunity and the two emerging human coronaviruses, which may provide new strategies for antiviral development (Wong et al., 2016). Similar studies on the innate immunity response in chickens against avian infectious bronchitis virus is reported by $\mathrm{He}$ et $a l$. in this special issue (He et al., 2016). The perspective authored by Liu et al. proposed strategies to prevention and control HCoVs from an- 
imal origins, and emphasized the "One Health" approach (Liu et al., 2016).

Since its first documented case in Saudi Arabia, MERS-CoV infection has been reported throughout the Middle East countries and transmitted to at least 12 other countries through travelling. Evidences dem-onstrated that MERS-CoV is transmitted to humans through direct exposure to dromedary camels. The article authored by Banik et al. analyzed the risk factors for mortality and disease severity among MERS patients and found that people with certain co-morbid conditions (diabetes, hypertension, renal disease, malignancy, miscellaneous conditions) are at particularly higher risk of dying or suffering severe outcome of MERS-CoV infection (Banik et al., 2016). Alqahtani et al. evaluated Australian pilgrims' knowledge and perceptions regarding the risk of MERS-CoV and camel contact at
Hajj, highlighting the importance for improved awareness among Hajj pilgrims and other travelers to the Middle East regarding MERS-CoV (Alqahtani et al., 2016).

\section{FOOTNOOTES}

We would like to thank all authors for their excellent contributions to this special issue.

\footnotetext{
$\triangle$ Correspondence:

Zheng-Li Shi:

Phone: +86-27-87197240,

Email: zlshi@wh.iov.cn

ORCID: 0000-0001-8089-163X

Deyin Guo:

Phone: +86-27-68752506,

Email:dguo@whu.edu.cn

ORCID: 0000-0002-8297-0814

Peter J. M. Rottier:

Phone: +31-30-2532462

Email: p.rottier@uu.nl

ORCID: 0000-0001-5373-732X
}

Published online: 19 February 2016

\section{REFERENCES}

Alqahtani AS, Wiley KE, Tashani M, et al. 2016. Virol Sin, 31: 89-93.

Banik GR, Alqahtani AS, Booy R, et al. 2016. Virol Sin, 31: 81-84.

ChenY, Guo D. 2016. Virol Sin, 31: 3-11.

Ge X, Wang N, Zhang W, et al. 2016. Virol Sin, 31: 31-40.

He Y, Xie Z, Dai J, et al. 2016. Virol Sin, 31: 57-68.

Liu L, Wang T, Lu J. 2016. Virol Sin, 31: 94-99.

Niu P, Shen J, Zhu N, et al. 2016.Virol Sin, 31: 85-88.

Wang H, Xue S, Yang H, et al. 2016. Virol Sin, 31: 24-30.

Wang J, Deng F, Ye G, et al. 2016. Virol Sin, 31: 49-56.

Wang M, Zhang W, Gao Y, et al. 2016. Virol Sin, 31: 78-80.

Wong LR, Lui PY, Jin DY. 2016. Virol Sin, 31: 12-23.

Xu L, Zhang F, Yang W, et al. 2016. Virol Sin, 31: 69-77.

Yip CC, Lam CS, Luk HK, et al. 2016.Virol Sin, 31: 41-48. 